\title{
Review of Congestion Management Methods for Distribution Networks with High Penetration of Distributed Energy Resources
}

\author{
Huang, Shaojun; Wu, Qiuwei; Liu, Zhaoxi; Nielsen, Arne Hejde
}

Published in:

Proceedings of 2014 ISGT Europe

Link to article, DOI:

10.1109/ISGTEurope.2014.7028811

Publication date:

2014

Link back to DTU Orbit

Citation (APA):

Huang, S., Wu, Q., Liu, Z., \& Nielsen, A. H. (2014). Review of Congestion Management Methods for Distribution Networks with High Penetration of Distributed Energy Resources. In Proceedings of 2014 ISGT Europe (pp. 16). IEEE. https://doi.org/10.1109/ISGTEurope.2014.7028811

\section{General rights}

Copyright and moral rights for the publications made accessible in the public portal are retained by the authors and/or other copyright owners and it is a condition of accessing publications that users recognise and abide by the legal requirements associated with these rights.

- Users may download and print one copy of any publication from the public portal for the purpose of private study or research.

- You may not further distribute the material or use it for any profit-making activity or commercial gain

- You may freely distribute the URL identifying the publication in the public portal 


\title{
Review of Congestion Management Methods for Distribution Networks with High Penetration of Distributed Energy Resources
}

\author{
Shaojun Huang, Qiuwei Wu, Zhaoxi Liu, Arne Hejde Nielsen \\ Centre for Electric Power and Energy, \\ Department of Electrical Engineering \\ Technical University of Denmark \\ Kgs. Lyngby, 2800 Denmark \\ shuang@elektro.dtu.dk, qw@elektro.dtu.dk, zhliu@elektro.dtu.dk, ahn@elektro.dtu.dk
}

\begin{abstract}
This paper reviews the existing congestion management methods for distribution networks with high penetration of DERs documented in the recent research literatures. The congestion management methods for distribution networks reviewed can be grouped into two categories - market methods and direct control methods. The market methods consist of dynamic tariff, distribution capacity market, shadow price and flexible service market. The direct control methods are comprised of network reconfiguration, reactive power control and active power control. Based on the review of the existing methods, the authors suggest a priority list of the existing methods.
\end{abstract}

Index Terms--Congestion management, direct control methods, distribute energy resources, distribution networks, market methods.

\section{INTRODUCTION}

Congestion in distribution networks that might occur caused by the high penetration of distributed energy resources (DERs), such as photovoltaic systems (PV systems), wind power systems (WPS), Electric Vehicle (EV) and heat pump (HP) is a big challenge to the grid planners and operators. Congestion problems in distribution networks are envisaged as voltage problem (bus voltage is close to or exceeding the limit, typically $+/-10 \%$ ) and overloading problem (loading is close to or exceeding the thermal limit of the power components).

The goal of the distribution network is to efficiently deliver the active power $P_{d}$ (shown in Fig.1) to the customers as required with high reliability. The customers can absorb some reactive power (e.g. induction motor) in the allowed range (according to the grid code). Deliver active and reactive power through a feeder will lead to voltage drop problems. Similarly, in the case of receiving renewable energy production, $P_{d}$ can be negative and it can lead to over-voltage problems. In both cases, if the power flow exceeds the thermal

The work is supported by the EU Seventh Framework Programme (FP7)

\footnotetext{
through the 'Ideal Grid for All (IDE4L)' project.
}

limits of power components, it will lead to overloading problems.

To solve (or alleviate) the under-voltage or over-voltage issues and overloading issues, distribution system operators (DSOs) can reinforce the distribution network (i.e. use cables/lines with higher current carrying capability and smaller impedance). The DSO can also change the total active and reactive power at bus 2 by installing local new distributed generators (DG, e.g. gas turbine) and FACTS devices, such as static VAR compensator (SVC), or by motivating the customers to change $P_{d}$ and $Q_{d}$ via market methods or directly controlling $P_{d}$ and $Q_{d}$ under pre-agreements with customers.

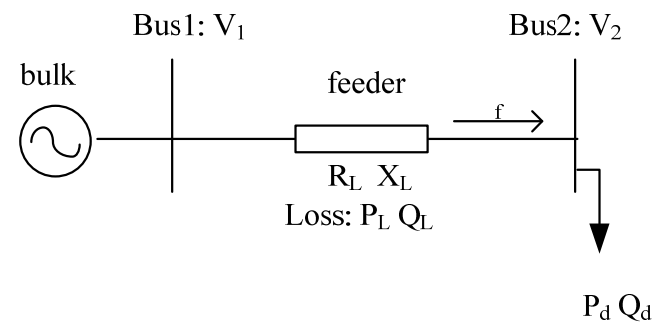

Fig. 1. illustration of a simple distribution grid.

This paper is to review the existing congestion management methods for distribution networks with high penetration of DERs documented in the recent research literatures. All of these methods are using one or more of the above mentioned methods plus some optimization methods to solve the congestion problem and achieve some other objectives (e.g. minimize costs or maximize social welfares). The methods reviewed in this paper cover the methods that have a central controller or a central organizer (e.g. DSO) in order to be able to perform the optimization methods. Therefore, those local methods having no optimization methods, such as voltage droop charging [1] and distributed 
reactive power generation control [2] through controllers embedded in the PV systems or EVs, are not covered.

Long term methods, such as grid reinforcement or installing new DG and FACTS [3], [4] will not be discussed either. However, it is worth mentioning that the cost of the long term methods lay down a maximum limit of the cost of the short term methods. Short term refers to the time frame from day-ahead to the real operation time.

The paper is organized as follows. The market methods or indirect control methods are discussed in section II. The direct control methods are described in section III. The discussion and comparison of all the direct and indirect methods reviewed are presented in section IV. In the end, a conclusion is drawn.

\section{INDIRECT CONTROL METHODS FOR CONGESTION MANAGEMENT}

Indirect control methods refer to market methods, which use price signals or contracts instead of direct orders to influence the behavior of the flexible demands (renewable energy sources (RES) are generally not responsive to the price signals because they are nearly cost free when produce electric energy). The methods developed in recent literatures include: day-ahead dynamic tariff (DT)[5]-[7], distribution capacity market[8], [9], intra-day shadow price[10] and flexibility service market[11]. The details of these methods will be reviewed in this section.

In the rest of this paper, the "aggregator" concept is used to represent the EVs or HPs in the distribution networks. Each EV or HP owner will subscribe his EV or HP to one aggregator and there will be several aggregators in one distribution network to provide the owners multiple choices and encourage market competition.

\section{A. Day-ahead Dynamic Tariff}

In this method, the flexible demands are price-sensitive demands and the DSO will find a theoretically lowest DT (time-varying) that would cause these flexible demands plus the basic loads (non-flexible demands) lower than the grid component loading limits.

A bi-level optimization model [7]was formulated to find the optimal DT:

$$
\begin{array}{cc} 
& \min \sum_{t \in T} c_{t} \\
\text { s.t. } & c_{t} \geq 0 \forall t \in T \\
& f_{t} \leq f^{\max } \forall t \in T \\
\text { s.t. } \quad & \min \sum_{t \in T} \beta_{t} \tilde{p}_{i, t}{ }^{T} \tilde{p}_{i, t}+\left(c_{t}+\alpha_{t}\right) 1^{T} \tilde{p}_{i, t} \\
& \tilde{p}_{i}^{\min } \leq e_{i, t} \leq e_{i}^{\max }, t \in T
\end{array}
$$

This model is exactly reflecting the above concept of the lowest DT, where: $c_{t}$ is the DT for time $t$ (e.g. from 0 till 23 clock of the next day)

$f_{t} \in R^{n_{L}}$ is the line loading at time $t, n_{L}$ is the number of distribution grid lines, $f_{t}$ can be computed from the flexible demands $\tilde{p}_{i, t} \in R^{m_{i}}$ and the non-flexible demands $\bar{p}_{i, t} \in R^{m_{i}}$

$\alpha$ is the predicted baseline spot price, $\beta$ is the sensitivity parameter (the total spot price is the baseline price plus an additional part which is sensitive to the amount of the flexible demands)

$e_{i, t} \in R^{m_{i}}$ is the status of the stored energy of the flexible demand, it can be calculated from the previous status, the flexible demands $\tilde{p}_{i, t}$ and the energy usage $u_{i, t}$,

$n_{B}$ is the number of aggregators (or balance responsible parties (BRP))

$m_{i}$ is the number of customers of aggregator $i$,

$T$ is a set including the next few hours.

This model is taken from [7] with some modifications to be more closely reflecting the above concept and respecting the situation of multiple aggregators. The bi-level optimization problem is generally hard to solve.

In the literature [5], the authors introduced the concept of “distribution locational marginal price (DLMP)". The DLMP is an extension of DT: the former could have different tariffs at different nodes in the same distribution grid where the latter has only one tariff in the whole distribution grid. Therefore, the DLMP is more flexible and could lead to better solution to the optimization problem than the DT, meaning lower total cost of customers.

According to [5], computing DLMP is also easier than solving the above bi-level problem: one-level optimization is enough and the DLMP is the Lagrange multipliers of the corresponding constraints of the optimization problem.

\section{B. Distribution Grid Capacity Market}

In this method, the capacity of distribution grid is allocated to the aggregators with an optimized price.

The distribution capacity market has been described in[7], [8], the market process is explained as follows:

- Step 1, the DSO sends an initial network tariff (normally it is zero) to aggregators

- $\quad$ Step 2, the aggregators individually perform their own optimization with the network tariff and communicate their charging schedules to the DSO.

- Step 3, the DSO evaluates whether the network capacity (distribution grid line loading) constraints are respected. If not, it raises the network tariff by a small amount during the moments when network capacity is exceeded. It sends the new tariff to aggregators and Go to step 2. If yes, go to next step. 
- Step 4, this procedure is converged, resulting in a certain grid tariff and a binding charging schedule of each aggregator (or the maximum capacity allocated to the aggregator of each moment)

With the allocated maximum distribution grid capacity, the aggregators can send their bids to the spot market.

The mathematic model to describe the above procedure can also be found in [7], which is modified and shown as follows:

- $c^{(0)}=0$,

- $\quad$ solve (4) individually by the aggregators, report $\tilde{p}_{i, t}$ to DSO

- DSO calculates $f^{(k)}$ based on $\tilde{p}_{i, t}$ and the predicted $\bar{p}_{i, t}$,

- $\quad c^{(k+1)}=c^{(k)}+\gamma \max \left(0, f^{\max }-f^{(k)}\right)$,

- $\quad$ stop if $\left|c^{(k+1)}-c^{(k)}\right| \leq \varepsilon$.

In [9], the authors have introduced an alternative method to obtain the grid tariff and the binding charging schedule. It is, however, more complicated, hence the details are not included here.

\section{Intra-day Shadow Price}

Literature [10] introduced a congestion prevention method via shadow price. Because the time-frame of this method is tens of minutes before operation time, the "intra-day" term is used to distinguish this method from the above day-ahead methods.

One hour or tens of minutes before operation time, the aggregators (or BRP) have already known the spot price and also have to try to stick to their demand plan according to their bids, otherwise a balance price will be charged. However, as approaching the operation time, the real demands could be different from the plan submitted to the spot market. Aggregators could use their flexible demands to mitigate these differences / imbalances, hence reducing the balance cost being charged. Therefore a new optimal schedule of the next few hours will come up, because they can have a rather precise prediction of the future demands compared to the dayahead one.

Assume the aggregators have already obtained some distribution capacity before the spot market, but now some of them need more and the others need less due to their renewed optimal schedules. Even though they might need more capacity at the same time, they value the additional capacity differently. Therefore there are needs of trading their capacity with a reasonable price (shadow price). This could be accomplished with the help of the DSO, who pursues no profit of this market but acts as a market operator.

The aggregators could choose their own optimization methods, according to the anticipated balancing price for imbalance. The authors of [10] use the following optimization method to illustrate their idea:

$$
\begin{array}{cc}
\min & \sum_{i \in N_{B}, t \in T}\left(1^{T}\left(\bar{p}_{i, t}+\tilde{p}_{i, t}\right)-q_{i, t}\right)^{2} \\
\text { s.t. } & e_{i}^{\min } \leq e_{i, t} \leq e_{i}^{\max }, \forall i \in N_{B}, t \in T \\
& \tilde{p}_{i}^{\min } \leq \tilde{p}_{i, t} \leq \tilde{p}_{i}^{\max }, \forall i \in N_{B}, t \in T \\
& f_{t} \leq f^{\max }, t \in T
\end{array}
$$

where $q_{i, t}$ is the total scheduled demands (of aggregator $i$ at $t$ ) according to the spot market.

Due to the coupling constraint (the last one in (5)), it can only be solved in a centralized manner, i.e. can only be solved by the DSO. In order to protect the private information of the aggregators, the authors of [10] proposed an iterative method to solve the problem in a distributed manner.

The optimization problem of aggregator $i$ becomes:

$$
\begin{gathered}
\min \sum_{t \in T}\left(1^{T}\left(\bar{p}_{i, t}+\tilde{p}_{i, t}\right)-q_{i, t}\right)^{2}+\lambda_{t}^{T} v_{i, t} \\
\text { s.t. } \quad e_{i}^{\min } \leq e_{i, t} \leq e_{i}^{\max }, t \in T \\
\\
\tilde{p}_{i}^{\min } \leq \tilde{p}_{i, t} \leq \tilde{p}_{i}^{\max }, t \in T
\end{gathered}
$$

where

$\lambda_{t} \in R^{n_{L}}$ is the shadow price,

$v_{i, t} \in R^{n_{L}}$ is the partial power flow due to the aggregator $i$,it can be calculated by $\tilde{p}_{i, t}$ and $\bar{p}_{i, t}$.

As documented in [10], the shadow price and the new optimal schedule are determined by the following iterative methods:

- $\lambda^{(0)}=0$

- $\quad$ solve (6) individually by the aggregators, report $\tilde{p}_{i, t}$ to DSO

- $\quad$ DSO calculates $f^{(k)}$ based on $\tilde{p}_{i, t}$ and $\bar{p}_{i, t}$

- $\quad \lambda^{(k+1)}=\lambda^{(k)}+\gamma\left(f^{\max }-f^{(k)}\right)$

- $\quad$ stop if $\left|\lambda^{(k+1)}-\lambda^{(k)}\right| \leq \varepsilon$.

Although (6) is a multi-period optimization problem, only the first period, i.e. $\tilde{p}_{i, 1}$, is actually implemented in real time operation. The optimization problem will roll over horizontally from one period to the next period. In this way, the shadow price and the renewed demand schedule can be obtained for each period. The aggregators need to pay the shadow price in addition to the spot price and the tariff (DT, DLMP or the one from the distribution capacity market). It can be further concluded that the DSO will not earn profit from the shadow price because the extra costs caused by shadow price are only among the aggregators (i.e. the sum is zero). 


\section{Flexibility service market}

Different from the above mentioned methods, the authors of [11]proposed a new option to solve the congestion: FLECH- flexibility service clearing house. The aggregators do not need to buy the distribution grid capacity, i.e. they can make their own demand plan without considering the distribution grid limitations. Instead, the DSO needs to buy the flexibility services to solve the congestion problem, e.g. buy a service which is to reduce the demands at a certain time and a certain location.

The aggregators can participate in the market based on their own benefits. They can sell some flexibility services to the market if they have such flexibility and it is economically beneficial to them. The DSO will decide whether to buy the flexibility services from the market or reinforce their grids.

The possible flexibility services, as mentioned in [11], include Flexibility Service of Overload Planned (FSOP), Flexibility Service of Overload Urgent (FSOU), Flexibility Service of Overload Reserve (FSOR), Flexibility Service of Overload Cap (FSOC) and Flexibility Service of Overload Maximum (FSOM).

- $\quad$ FSOP will be activated before the overloading (e.g. $70 \%$ of the maximum loading limit) time.

- $\quad$ FSOU will be activated exactly when the overloading appears.

- $\quad$ FSOR will be activated sharply when the line loading hits the maximum limit (i.e. 100\%) or there is a fault at the neighboring feeder and the line loading is above $70 \%$.

- $\quad$ FSOC promises a feeder capacity limit specified by the DSO (e.g. 70\%) will not be violated.

- $\quad$ FSOM means that the Aggregators have the obligation to guarantee that their local portfolio will not exceed a certain limit (e.g. 70\%) specified by the DSO.

The flexibility service market is working in parallel with the conventional markets, such as the spot market, the intraday and intra-hour market. Issues like how to optimally make the flexibility services, how to fulfil the demands of the customers after the services are activated (e.g. the batteries still need to be charged before a predefined time), however, are not mentioned by the authors of [11].

\section{DiRECT CONTRL METHODS FOR CONGESTION MANAGEMENT}

Though the market methods can solve the congestion very effectively in many cases, there are some cases where they can only partly solve the congestion due to market failures or forecasting errors. In such cases, direct control methods should be employed and served as a backup against the system service interruption due to the overloading or over/under voltage.

The direct control methods for congestion management for distribution networks with high penetration of DERs consist of network reconfiguration, reactive power control and active power control.

\section{A. Reconfiguration}

Reconfiguration in distribution networks refers to the change of the grid structure through changing the status of the normal-open switches and some normal-close switches in order to maintain the same radial structure but more efficient or suitable for delivering the power to the customers.

The optimization problem:

$$
\begin{array}{ll}
\min _{y} o(f) \\
\text { s.t. } & h(f, v)=0 \\
& g(f, v, y) \leq 0
\end{array}
$$

is a typical reconfiguration problem, where

$f$ is the line loadings

$v$ is the bus voltages

$y$ is the status of the switches, integer variable

$o$ is the objective function, e.g. line losses

$h$ is load flow equations

$g$ is inequalities regarding the voltage limits, line loading limits, radial structure assurance and the relation between the line loading $f$ and the switch status $y$.

For solving the congestion problems, $o$ can be any function (e.g. a dummy function), as long as (7) is feasible, the congestion is solved without any cost (from the customer point of view, reconfiguration is cost free). But DSO would rather to choose to maximize a kind of welfare, e.g. minimizing the total line losses or balancing the feeder loadings.

Problem (7) is a nonlinear integer optimization problem, which is generally hard to solve. There are some methods developed in recent literatures to solve this problem:

- $\quad$ Mixed integer linear program (MILP) method [12]. The problem can be linearized to a MILP problem and is solved using conventional MILP solvers.

- $\quad$ LP or NLP methods[13], [14]. The integer part of the problem is removed and incorporated into the solvers (or the solving process) of the LP or NLP problem. (a) For instance, the authors of [14]developed a method called 'branch exchange method'. This method starts from a feasible point (with respect to $y$ ) and then move to the next point that reduces the cost function most among all the neighboring (changing only one pair of switches) feasible points. The neighboring feasible points are identified by the so called branch exchange method. At last, the search process is ended at a local minimum point. (b) As another example, the authors of [13]developed 'modified simplex method'. In order to use the simplex method, the problem is formulated as a linear problem by relaxing the model. The simplex method was modified to ensure that each step of exchanging basic and non-basic variables will maintain a radial structure. A disadvantage of these two methods is that the global minimum point might not be found. 
- Heuristic methods, e.g. adapted genetic algorithm (GA) method developed in[15], [16]. This family of methods can generally solve the nonlinear integer problems efficiently and tend to reach the global minimum, but not guaranteed due to the nondeterministic nature of these methods.

\section{B. Reactive Power Control}

Reactive power support from flexible AC transmission system (FACTS) devices (e.g. Static VAR compensator (SVC)) and converter interfaced flexible demands (e.g. EV) or RER (e.g. PV) can relieve the under-voltage (or over-voltage) problem, especially in weak networks (long feeders), where voltage problems are more critical than thermal problems (line loading limit).

Reactive power control problem can be formed as,

$$
\begin{array}{ll}
\min _{Q_{d}} o(f) \\
\text { s.t. } & h\left(f, v, Q_{d}\right)=0 \\
& g\left(f, v, Q_{d}\right) \leq 0
\end{array}
$$

where $Q_{d}$ is the adjustable reactive power from the support devices. It can be positive (to mitigate voltage rise) or negative (to mitigate voltage drop). Those non-adjustable reactive power demands are parameters in the load flow.

Reactive power is nearly cost free, if the losses of the devices due to the increase of the apparent power are negligible. Therefore, as long as problem (8) is feasible, the congestion is solved. The reactive power dispatch scheme found by (8) is generally better than the constant power factor scheme (i.e. the reactive power is determined by the active power of the same device locally) with respect to the loss reduction [17]. Although the amount of the deduction is normally marginal, as stated in [17], congestion problem is solved. Authors of [18] suggest that on-load tap changer (OLTC) should be employed together with the reactive power control and therefore solve the congestions more efficiently.

\section{Active Power Control}

Different from the above two nearly free methods (see sub section A and B), the active power control method is associated with costs or discomfort to the customers. It is assumed that the congestion is only caused by the RER or flexible demands. Therefore the active power control method can solve the congestion efficiently; the point lies in how to minimize the cost or dissatisfaction.

The active power control can be stated as an optimization problem:

$$
\begin{array}{ll}
\min _{\Delta P_{d}} & o\left(f, \Delta P_{d}\right) \\
\text { s.t. } & h\left(f, v, \Delta P_{d}\right)=0 \\
& g\left(f, v, \Delta P_{d}\right) \leq 0
\end{array}
$$

where $\Delta P_{d}$ is the adjustment of the active power (also known as the amount of load shedding, generator curtailment, etc.).
Regarding the selection of the objective function:

- In [19], the authors use the summation of all the adjustments and the line losses as the objective function and claim that it is suitable for the curtailment of the renewable energies, since the generation cost is nearly free and therefore the same for different customers.

- in [20], different from the previous one, individual weights assigned to all customers are used in the summation, which in turn, makes the problem suitable for active power control of flexible demands, in addition to renewable energies as in the previous one. How to decide the weights for different customers is not discussed by the authors of [20].

- $\quad$ in [21], the up and down regulation prices in the pool based electricity market are used as the weights of the up adjustments (negative) and down adjustments (positive) respectively in the summation of the objective function, where the line losses are not considered. In this way, the congestion costs, i.e. the deviations from the preferred transaction schedules of the customers, are minimized.

DSO should choose the objective function according to the actual situation, e.g. the cost of the generators, the agreement with the customers and the other market conditions.

\section{DISCUSSIONS}

\section{A. Summary of The Methods}

The responsible parties (or actors), relation to the conventional market and time frame, the objective function (the cost function) of the above discussed congestion management methods are summarized in Table I.

\section{B. Priority Discussions}

The question about in which priority DSO should use the above discussed methods is still open. However, as an example, the authors suggest a priority scheme as the follows.

- Firstly, the cost free (or nearly cost free) methods should be employed, such as reconfiguration and reactive power control methods. DSO should use these two methods to solve the congestion problem as much as possible. In addition, DSO's own assets, such as online tap-changers of transformers and voltage regulators, should be considered as well since they are also nearly cost free.

- Secondly, if the above methods cannot solve the congestion problem completely, market methods should be employed. DSO and aggregators should maximize their own welfare by participating in the markets.

- At last, if all the above methods are exhausted, active power control method should be used as the last defense before the relays black out a whole feeder. 
TABLE I

SuMmery OF THE CONGESTION MANAgEMENT Methods

\begin{tabular}{|c|c|c|c|}
\hline Method & Responsible parties & $\begin{array}{c}\text { Relation to the } \\
\text { conventional market/ } \\
\text { time frame }\end{array}$ & Objective \\
\hline DT & only DSO & before spot market & lowest DT, that could prevent congestion \\
\hline $\begin{array}{l}\text { Distribution } \\
\text { market }\end{array}$ & DSO and aggregators & before spot market & lowest tariff, that could prevent congestion \\
\hline Intra-day shadow price & $\begin{array}{l}\text { DSO and aggregators, but } \\
\text { DSO has no profit }\end{array}$ & $\begin{array}{l}\text { after spot market, tens of } \\
\text { minutes before operation }\end{array}$ & lowest imbalance \\
\hline Flexibility service market & $\begin{array}{l}\text { DSO and aggregators, but } \\
\text { aggregators are not obliged }\end{array}$ & $\begin{array}{l}\text { parallel to the conventional } \\
\text { market }\end{array}$ & not mentioned \\
\hline Reconfiguration & \multirow{3}{*}{ only DSO } & \multirow{3}{*}{$\begin{array}{l}\text { it can be either a day-ahead } \\
\text { planning or a real-time } \\
\text { operation }\end{array}$} & minimize line losses, balance line loadings, etc. \\
\hline Reactive power control & & & minimize line losses, maximize loadability, etc. \\
\hline Active power control & & & $\begin{array}{l}\text { minimize the adjustments, minimize the cost of } \\
\text { adjustments, etc. }\end{array}$ \\
\hline
\end{tabular}

\section{CONCLUSIONS}

The paper provides a review of most of the existing congestion management methods for distribution networks with high penetration of DERs, including market methods and direct control methods. The market methods consist of dayahead dynamic tariff, distribution capacity market, shadow price and flexibility service market. The market methods can generally alleviate the congestion by influencing the DERs. However, it is important to have the direct control methods as a backup for the market methods which are distribution network reconfiguration, reactive power control and active power control. The summary and discussion of these methods have been given in order to give a suggestion of selecting the appropriate methods to solve the congestion problem.

\section{REFERENCES}

[1] S. Huang, J. R. Pillai, B. Bak-Jensen, and P. Thogersen, "Voltage support from electric vehicles in distribution grid," in Proc. 15th European Conference on Power Electronics and Applications (EPE), IEEE, 2013, pp. 1-8.

[2] P. M. S. Carvalho, P. F. Correia, and L. A. F. Ferreira, "Distributed reactive power generation control for voltage rise mitigation in distribution networks,” IEEE Trans. Power Syst., vol. 23, no. 2, pp. 766-772, May 2008.

[3] S. Ganguly, "Multi-objective planning for reactive power compensation of radial distribution networks with unified power quality conditioner allocation using particle swarm optimization," IEEE Trans. Power Syst., vol. PP, no. 99, pp. 1-10, 2014.

[4] M. Alonso, H. Amarís, C. Álvarez, and R. Albarracín, "Reactive power planning in distribution networks with distributed generation," in Proc. 7th Mediterranean Conference and Exhibition on Power Generation, Transmission, Distribution and Energy Conversion, IET, 2010, pp. 152-152.

[5] R. Li, Q. Wu, and S. S. Oren, "Distribution locational marginal pricing for optimal electric vehicle charging management,” IEEE Trans. Power Syst., vol. 29, no. 1, pp. 203-211, Jan. 2014.

[6] N. O’Connell, Q. Wu, J. Østergaard, A. H. Nielsen, S. T. Cha, and Y Ding, "Day-ahead tariffs for the alleviation of distribution grid congestion from electric vehicles,” Electr. Power Syst. Res., vol. 92, pp 106-114, 2012.

[7] R. A. Verzijlbergh, L. J. De Vries, and Z. Lukszo, "Renewable energy sources and responsive demand. Do we need congestion management in the distribution grid?,” IEEE Trans. Power Syst., pp. 1-10, 2014.

[8] P. Bach Andersen, J. Hu, and K. Heussen, "Coordination strategies for distribution grid congestion management in a multi-actor, multiobjective setting," in Proc. 3rd IEEE PES Innovative Smart Grid Technologies Europe (ISGT Europe), IEEE, 2012, pp. 1-8.
[9] J. Hu, S. You, M. Lind, and J. Ostergaard, "Coordinated charging of electric vehicles for congestion prevention in the distribution grid," IEEE Trans. Smart Grid, vol. 5, no. 2, pp. 703-711, Mar. 2014.

[10] B. Biegel, P. Andersen, J. Stoustrup, and J. Bendtsen, "Congestion management in a smart grid via shadow prices," in Proc. 8th IFAC Symposium on Power Plant and Power System Control, 2012, pp. 518523.

[11] C. Zhang, Y. Ding, N. Nordentoft, P. Pinson, and J. Østergaard, "FLECH-A danish market solution for DSO congestion management through DER flexibility services,” J. Mod. Power Syst. Clean Energy, vol. 2, no. 2, pp. 126-133, 2014.

[12] J. F. Franco, M. J. Rider, M. Lavorato, and R. Romero, “A mixedinteger LP model for the reconfiguration of radial electric distribution systems considering distributed generation," Electr. Power Syst. Res., vol. 97, pp. 51-60, Apr. 2013.

[13] A. Abur, "A modified linear programming method for distribution system reconfiguration,” Int. J. Electr. Power Energy Syst., vol. 18, no. 7, pp. 469-474, Oct. 1996.

[14] M. E. Baran and F. F. Wu, "Network reconfiguration in distribution systems for loss reduction and load balancing," IEEE Trans. Power Deliv., vol. 4, no. 2, pp. 1401-1407, Apr. 1989.

[15] B. Enacheanu, B. Raison, R. Caire, O. Devaux, W. Bienia, and N. Hadjsaid, "Radial network reconfiguration using genetic algorithm based on the matroid theory," in Proc. IEEE Power and Energy Society General Meeting - Conversion and Delivery of Electrical Energy in the 21st Century, IEEE, 2008, pp. 1-1.

[16] M. H. Shariatkhah and M. R. Haghifam, "Using feeder reconfiguration for congestion management of smart distribution network with high DG penetration," in Proc. CIRED Workshop: Integration of Renewables into the Distribution Grid, IET, 2012, pp. 316-316.

[17] K. Daroj and W. Limpananwadi, "Reactive power dispatch scheme evaluation for synchronous based distributed generators to reduce real power loss in distribution systems," in Proc. IEEE International Conference on Sustainable Energy Technologies, IEEE, 2008, pp. 1178-1183.

[18] F. A. Viawan and D. Karlsson, "Voltage and reactive power control in systems with synchronous machine-based distributed generation," IEEE Trans. Power Deliv., vol. 23, no. 2, pp. 1079-1087, Apr. 2008.

[19] Q. Zhou and J. W. Bialek, "Generation curtailment to manage voltage constraints in distribution networks," IET Gener. Transm. Distrib., vol. 1, no. 3, pp. 492 - 498, May 2007.

[20] A. R. Malekpour, A. R. Seifi, M. R. Hesamzadeh, and N. Hosseinzadeh, "An optimal load shedding approach for distribution networks with DGs considering capacity deficiency modelling of bulked power supply,” in Proc. Power Engineering Conference, IEEE, 2008, pp. 1-7.

[21] S. Balaraman and N. Kamaraj, "Application of differential evolution for congestion management in power system,” Mod. Appl. Sci., vol. 4, no. 8, pp. 33-42, Jul. 2010. 